\title{
One pion production in neutrino nucleon and neutrino nucleus interaction
}

\author{
L. Alvarez-Ruso \\ Instituto de Física Corpuscular (IFIC), Centro Mixto CSIC-Universidad de Valencia, Institutos \\ de Investigación de Paterna, Apartado. 22085, 46071 Valencia, Spain \\ E-mail: luis.alvarez@ific.uv.es \\ E. Hernández* \\ Departamento de Física Fundamental e IFFyM, Universidad de Salamanca, 37008 Salamanca, \\ Spain \\ E-mail: gajatee@usal.es
}

\section{J. Nieves}

Instituto de Física Corpuscular (IFIC), Centro Mixto CSIC-Universidad de Valencia, Institutos de Investigación de Paterna, Apartado. 22085, 46071 Valencia, Spain

E-mail: jmnieves@ific.uv.es

\section{J.E. Sobczyk}

Instituto de Física Corpuscular (IFIC), Centro Mixto CSIC-Universidad de Valencia, Institutos de Investigación de Paterna, Apartado. 22085, 46071 Valencia, Spain

E-mail: Joanna. Sobczykeific.uv.es

\section{M.J. Vicente Vacas}

Departamento de Física Teórica and Instituto de Física Corpuscular (IFIC), Centro Mixto Universidad de Valencia-CSIC, Institutos de Investigación de Paterna, 46071 Valencia, Spain E-mail: Manuel.J.Vicente@uv.es

\begin{abstract}
We very briefly describe the main ingredients, including the latest modifications, of the "HNV" model for one pion production in neutrino nucleon reactions. We present results for total cross sections as well as pion angular distributions with respect to the pion polar angles measured in the pion-nucleon center of mass. The latter show that the approximation of taking an isotropic distribution, as done in some Monte Carlo generators, is not supported by microscopic calculations. With the corresponding medium effects taken into account, the model is applied to one pion production in nuclei for which we show some preliminary results.
\end{abstract}

The 21st international workshop on neutrinos from accelerators (NuFact2019)

August 26 - August 31, 2019

Daegu, Korea

\footnotetext{
* Speaker.
} 


\section{Introduction}

In this contribution we present some results obtained with the latest version of the neutrino induced one-pion production model originally developed in Ref. [1]. In its primary form, the model, that has become to be known as the HNV model, contained the dominant $\Delta(1232)$ contribution (Figure 1 left) and non-resonant terms (Figure 1 center) that are required by chiral symmetry. Later on, and with the idea of extending the validity of the model to higher $W_{\pi N}$ invariant masses, we included in Ref. [2] the contribution from the $D_{13}(1520)$ resonance (see Figure 1 right), the one that apart from the $\Delta$ gives the largest contribution below $W_{\pi N}=2 \mathrm{GeV}$. In Ref. [3] the model was

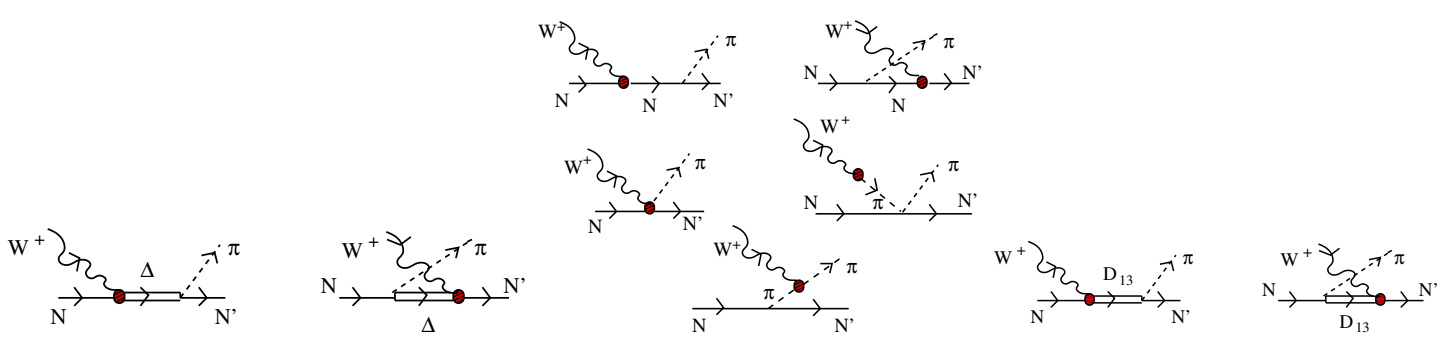

Figure 1: Pictorial content of the HNV model as it was introduced in Ref. [1] (left plus center), and the new contribution from the $D_{13}(1520)$ resonance (right) added in Ref. [2].

modified so that it complied with Watson's theorem. Splitting the amplitude between Direct Delta $\left(T_{\Delta}\right)$ and the rest $\left(T_{B}\right)$, we followed Olsson method [4] and modified our amplitude as

$$
T_{B}+T_{\Delta} \rightarrow T_{B}+e^{i \delta_{V}} T_{\Delta P}^{V}+e^{i \delta_{A}} T_{\Delta P}^{A}
$$

where the, $q^{2}$ and $W_{\pi N}$ depending, vector $\delta_{V}$ and axial $\delta_{A}$ Olsson phases were fixed by requiring that the dominant vector and axial multipoles with the $\Delta(1232)$ quantum numbers have the correct phase $\delta_{P_{33}}\left(W_{\pi N}\right)$. Finally, in Ref. [5], and inspired by the work of Ref. [6], we included the latest modification that affected the spin-1/2 part of the Rarita-Schwinger projector present in the $\Delta$ propagator. The idea behind this modification was to obtain a better reproduction of the $v_{\mu} n \rightarrow \mu^{-} n \pi^{+}$ channel, a channel that is very sensitive to the crossed- $\Delta$ term and thus to the spin- $1 / 2$ part of the $\Delta$ propagator. The interested reader can find all the details of the model in the above given references and references therein.

\section{Results at the nucleon level}

In Fig. 2 we show results for total cross sections for $v_{\mu}$ induced reactions taken from Ref. [7]. Since the data has been measured in deuterium, we take into account deuteron effects as explained in Ref. [8]. We compare our results with the theoretical predictions of the more elaborate, fullyunitary, dynamical coupled-channel (DCC) model of Ref. [9] and experimental data from Refs. [10, 11, 12]. The agreement with data is good. We also find a good agreement with the DCC model predictions. The largest discrepancy with the DCC model occurs for the $v_{\mu} n \rightarrow \mu^{-} n \pi^{+}$case for which we give a better global description due to our modification of the $\Delta$ propagator. 

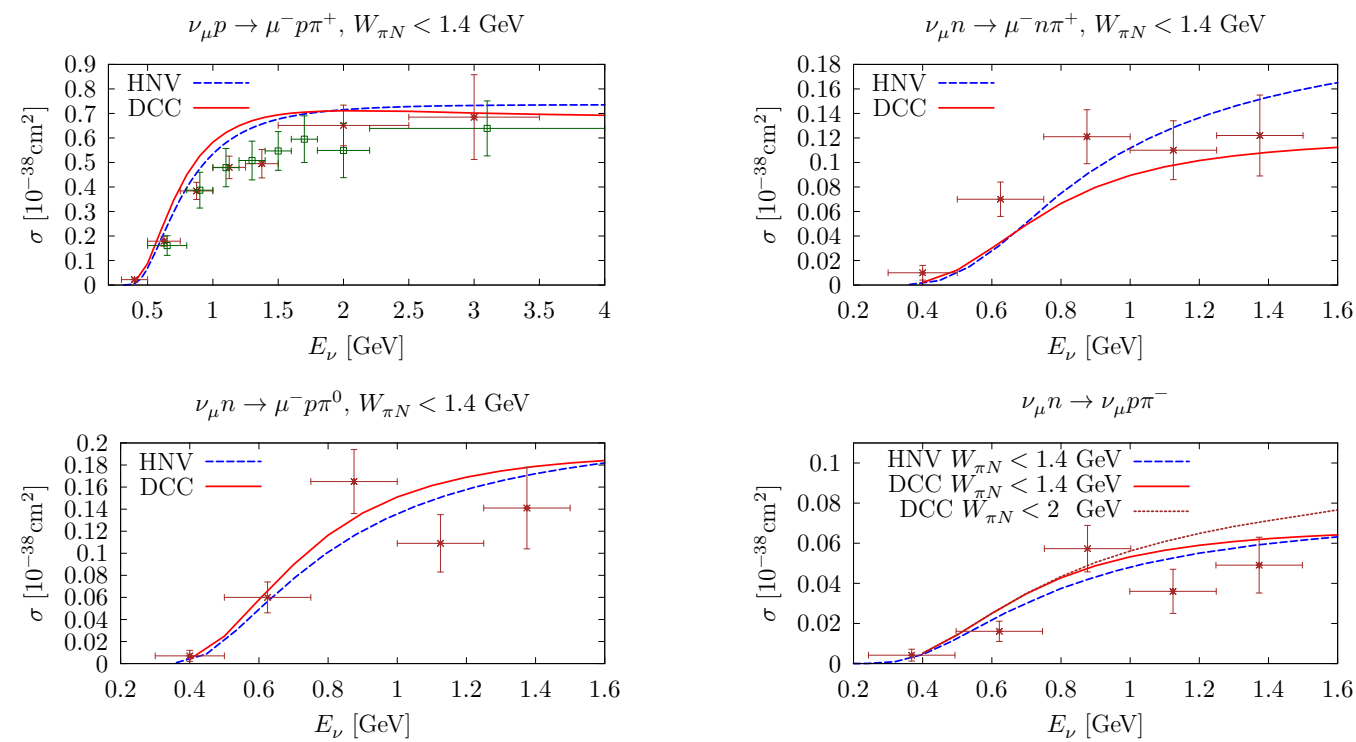

Figure 2: Different $v_{\mu} N \rightarrow \rightarrow \mu^{-} N^{\prime} \pi$ total cross sections as a function of the neutrino energy. We compare our results (HNV) with the predictions by the DCC model [9] and experimental data. The latter have been taken from the reanalysis done in Refs. [10,11] of old ANL (crosses) and BNL (open squares) data, except for the $v_{\mu} n \rightarrow v_{\mu} p \pi^{-}$reaction that we take from Ref. [12].

In Fig. 3 we show results for $d \sigma / d \Omega_{\pi}^{*}$, with $\Omega_{\pi}^{*} \equiv \theta_{\pi}^{*}, \phi_{\pi}^{*}$ the pion polar angles measured in the final nucleon-pion center of mass. As seen, the distributions are clearly anisotropic and channel dependent. Similar results are found for the DCC model (see Ref. [7]). This is in contrast to the approximation in some Monte Carlo generators where an isotropic channel-independent distribution is taken. Besides, and as explained in Ref. [7], the lack of reflection symmetry with respect to the $\phi_{\pi}^{*}=0$ line, clearly seen in some of the panels, is a consequence of parity violation and it originates from the interference between multipoles with different quantum numbers that are present in the amplitude.
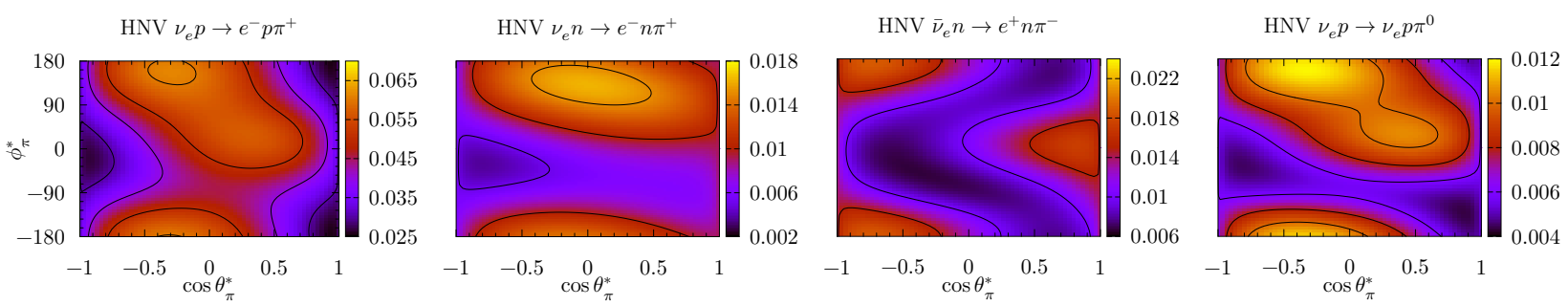

Figure 3: HNV model prediction for $d \sigma / d \Omega_{\pi}^{*}$ differential cross section in units of $10^{-38} \mathrm{~cm}^{2}$, as a function of $\phi_{\pi}^{*}$ and $\theta_{\pi}^{*}$, evaluated at $E_{V}=1 \mathrm{GeV}$ and with a $W_{\pi N}<1.4 \mathrm{GeV}$ cut.

\section{Results at the nucleus level}

The in-medium modification of our pion production model at the nucleon level, as well as details on the Monte Carlo code we use to evaluate the final state interaction effects are discussed 
in Ref. [2] and references therein. Here, we show some preliminary results, obtained with the latest modifications of the model, that we compare to theoretical results by the Giessen Group (GiBUU in the Figure) [13] and experimental data from the MiniBooNE [14], MINERvA [15] and T2K [16] Collaborations. For MiniBooNE and T2K we have integrated the neutrino flux up to $2 \mathrm{GeV}$ neutrino energy, while in the case of MINER $v \mathrm{~A}$, where a cut $W_{\pi N}<1.4 \mathrm{GeV}$ is applied, we go up to $5 \mathrm{GeV}$. We get a reasonable description of MiniBooNE data but we over predict in the case of MINERvA, though we seem to get right the forward pion data. For T2K the agreement is reasonable considering the large errors but, compared to central values, we lack forward pions.
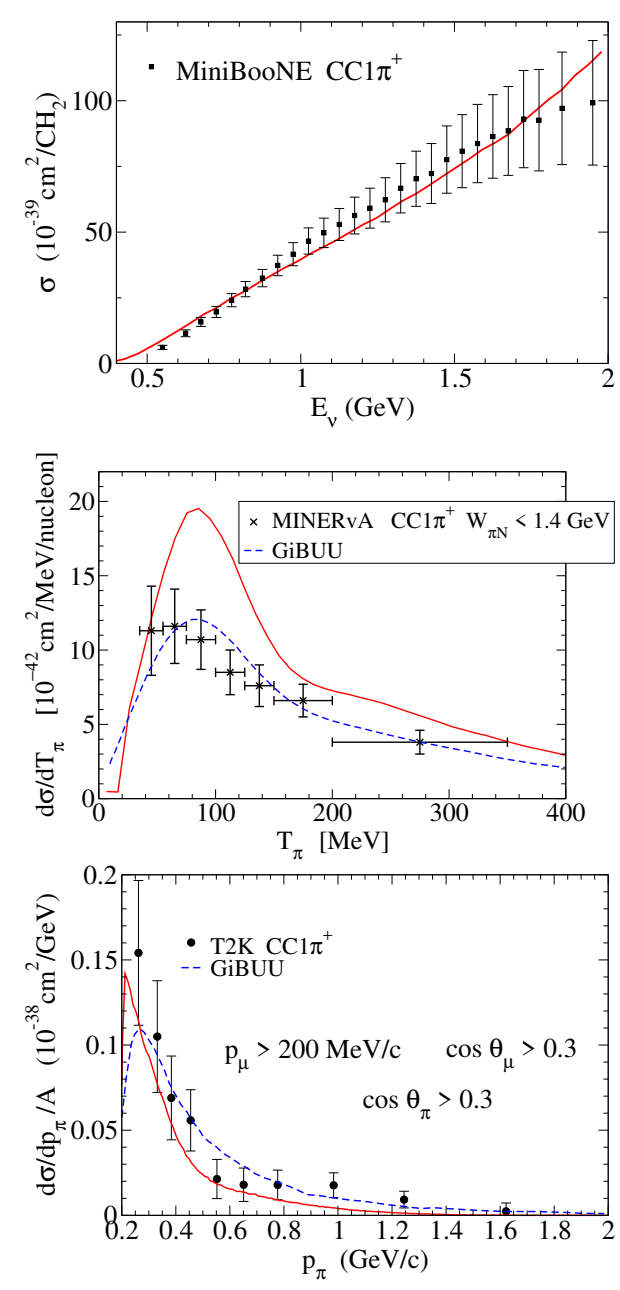
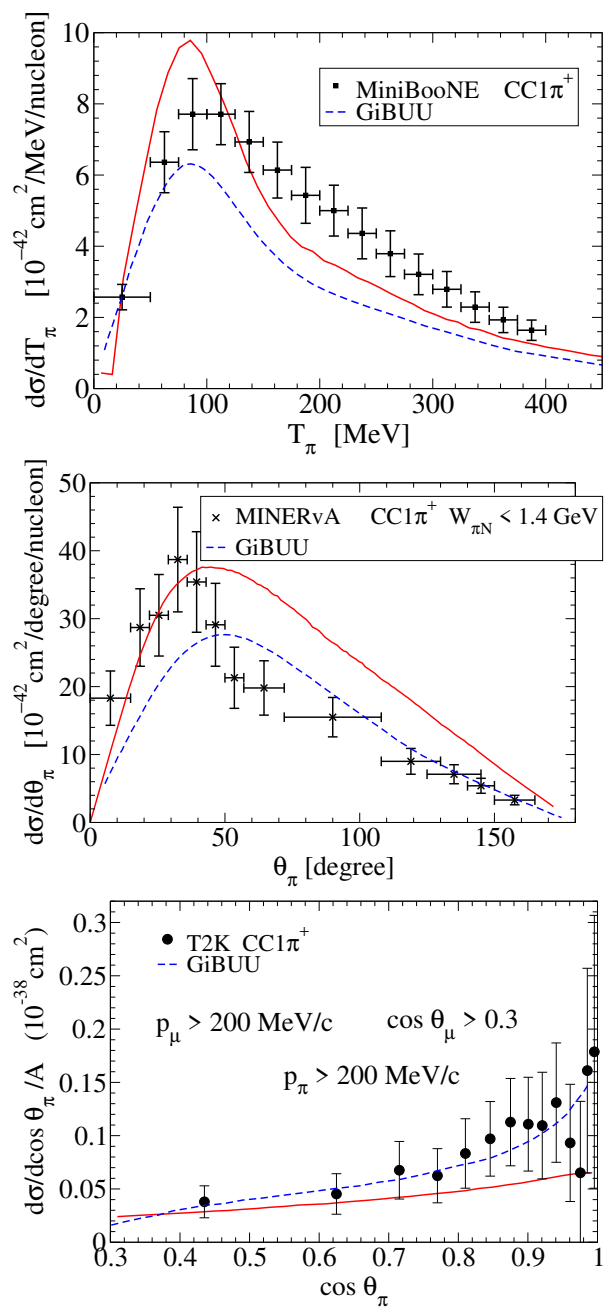

Figure 4: Results of our model (solid red), including the latest modifications at the nucleon level. We compare our results to experimental data by the MiniBooNE [14], MINERvA [15] and T2K [16] Collaborations. We also show the theoretical results obtained by GiBUU [13].

Research supported in part by the Spanish Ministerio de Economía y Competitividad (MINECO) and the European Regional Development Fund (ERDF) under contracts FIS2017-84038-C2-1-P, FPA2016-77177-C2-2-P, SEV-2014-0398 and by the EU STRONG-2020 project under the program H2020-INFRAIA-2018-1, grant agreement no. 824093. 


\section{References}

[1] E. Hernández, J. Nieves, and M. Valverde, Weak Pion Production off the Nucleon, Phys. Rev. D 76, 033005 (2007) [arXiv:hep-ph/0701149v2].

[2] E. Hernández, J. Nieves, M. J. Vicente Vacas, Single pion production in neutrino nucleus scattering, Phys. Rev. D 87, 113009 (2013) [arXiv:1304.1320v2 [hep-ph]].

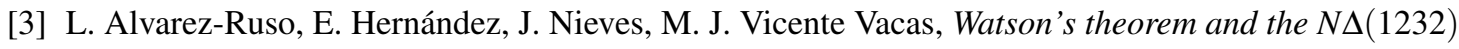
axial transition, Phys. Rev. D 93, 014016 (2016) [arXiv:1510.06266v3 [hep-ph]].

[4] M.G. Olsson, Solutions of the multichannel unitarity equations describing the addition of a resonance and background: Application to a pole model of photoproduction, Nuclear Physics B78, 55 (1974).

[5] E. Hernández, J. Nieves, Neutrino-induced one-pion production revisited: the $v_{\mu} n \rightarrow \mu^{-} n \pi^{+}$ channel, Phys. Rev. D 95, 053007 (2017) [arXiv:1612.02343v2 [hep-ph]].

[6] V. Pascalutsa, Correspondence of consistent and inconsistent spin - 3/2 couplings via the equivalence theorem, Phys. Lett. B 503, 85 (2001) [arXiv:hep-ph/0008026 [hep-ph]].

[7] J.E. Sobczyk, E. Hernández, S.X. Nakamura, J. Nieves, T. Sato, Angular distributions in electroweak pion production off nucleons: Odd parity hadron terms, strong relative phases, and model dependence, Phys. Rev. D 98, 073001 (2018) [arXiv:1807.11281 [hep-ph]].

[8] E. Hernández, J. Nieves, M. Valverde, and M. J. Vicente Vacas $N-\Delta(1232)$ axial form factors from weak pion production Phys. Rev. D 81, 085046 (2010) [arXiv:1001.4416 [hep-ph]].

[9] S. X. Nakamura, H. Kamano, and T. Sato, Dynamical coupled-channels model for neutrino-induced meson productions in resonance region, Phys. Rev. D92, 074024 (2015) [arXiv:1506.03403 [hep-ph]].

[10] P. Rodrigues, C. Wilkinson, and K. McFarland, Constraining the GENIE model of neutrino-induced single pion production using reanalyzed bubble chamber data, Eur. Phys. J. C76, 474 (2016) [arXiv:1601.01888 [hep-ex]].

[11] C. Wilkinson, P. Rodrigues, S. Cartwright, L. Thompson, and K. McFarland, Reanalysis of bubble chamber measurements of muon-neutrino induced single pion production, Phys. Rev. D90, 112017 (2014) [arXiv:1411.4482 [hep-ex]].

[12] M. Derrick et al., Study of the reaction $v n \rightarrow v p \pi^{-}$, Phys. Lett. 92B, 363 (1980); [Erratum: Phys. Lett. 95B,461(1980)].

[13] U. Mosel, and K. Gallmeister, Muon-neutrino-induced charged-current pion production on nuclei, Phys. Rev. C 96, 015503 (2017) [arXiv:1708.04528v3 [nucl-th]].

[14] A. A. Aguilar-Arevalo et al. (MiniBooNE Collaboration), Measurement of neutrino-induced charged-current charged pion production cross sections on mineral oil at $E_{V} \sim 1 \mathrm{GeV}$, Phys. Rev. D 83, 052007 (2011) [arXiv:1011.3572v3 [hep-ex]].

[15] B. Eberly et al. (MINERvA Collaboration), Charged pion production in $v_{\mu}$ interactions on hydrocarbon at $\left\langle E_{v}\right\rangle=4.0 \mathrm{GeV}$, Phys. Rev. D 92, 092008 (2015) [arXiv:1406.6415v3 [hep-ex]].

[16] K. Abe et al. (T2K Collaboration), First measurement of the muon neutrino charged current single pion production cross section on water with the T2K near detector, Phys. Rev. D 95, 012010 (2017) [arXiv:1605.07964v2 [hep-ex]]. 